INVESTIGACIÓN

https://doi.org/10.15198/seeci.2020.53.83-102

Recibido: 16/06/2020 --- Aceptado: 26/08/2020 --- Publicado: 15/11/2020

\title{
JUEGO DE VÍDEO A PARTIR DE LA METODOLOGÍA GAME-BASED LEARNING COMO MEDIO DE COMUNICACIÓN PARA LA FORMACIÓN DE HÁBITOS ALIMENTARIOS EN NIÑOS CON ENFERMEDAD CELÍACA
}

\section{VIDEO GAME BASED ON THE GAME-BASED LEARNING METHODOLOGY AS A MEANS OF COMMUNICATION FOR THE FORMATION OF EATING HABITS IN CHILDREN WITH CELIAC DISEASE}

Jeanín M. Santos Rivera': Universidad Metropolitana. Venezuela. jsantos@unimet.edu.ve

Elvira E. Navas Piñate²: Universidad Metropolitana. Venezuela. enavas@unimet.edu.ve

\section{RESUMEN}

En este estudio se propone el diseño de un videojuego basado en la metodología game-based learning como medio de comunicación para facilitar la formación de hábitos alimentarios saludables en niños con diagnóstico de Enfermedad Celiaca (EC). La necesidad surge de lo observado en la práctica profesional en la consulta de nutrición, donde madres de pacientes celiacos manifiestan como preocupación principal el hecho de que el niño, expuesto a diversas actividades sociales y académicas, en la cuales no necesariamente se encuentra con su compañía, sea capaz de cumplir la dieta libre de gluten, único tratamiento para la EC. La investigación se fundamenta en el concepto y manifestaciones clínicas de la EC, el uso del juego como recurso para la enseñanza (game-based learning), y la visión teórica del paradigma constructivista del aprendizaje. Por sus características, esta investigación resulta congruente con el Proyecto Factible, en un nivel descriptivo y se apoya en la investigación documental utilizando la técnica de análisis y el registro documental como instrumento para la obtención de información. Se desarrolló en

\footnotetext{
${ }^{1}$ Jeanín M. Santos Rivera: Licenciada en Nutrición y Dietética (UCV), Especialista en Tecnología, Aprendizaje y Conocimiento (UNIMET). Profesora asistente de la Universidad Metropolitana, Caracas, Venezuela, de las asignaturas de Arte y Ciencia de la Gastronomía, Educación Nutricional y Tecnologías para el Aprendizaje. Email: jsantos@unimet.edu.ve.

${ }^{2}$ Elvira E. Navas Piñate: Ingeniero en Computación (USB), Especialista en Informática Educativa (USB), Doctora en Investigación en Didáctica y Organización de Instituciones Educativas (US). Profesora titular de la Universidad Metropolitana, Caracas, Venezuela, de las asignaturas de la línea de Programación de Computadoras y en Tecnología Educativa. Decana de la Facultad de Ciencias y Artes. Email: enavas@unimet.edu.ve.
} 
Santos Rivera, J. M. y Navas Piñate, E. E. Juego de vídeo a partir de la metodología gamebased learning como medio de comunicación para la formación de hábitos alimentarios en niños con enfermedad celíaca

dos fases, una fase preparatoria donde se obtuvo la información documental necesaria para el cumplimiento de los objetivos propuestos y una fase de diseño que consistió en el desarrollo de los componentes instruccionales del videojuego y la aproximación a un prototipo. El videojuego diseñado es entonces un juego educativo (serious game) llamado El Mundo de Celia, que se compone de un mundo en forma de tablero, compuesto por tres niveles, donde el participante debe superar nueve retos en total, y mediante la práctica del juego, puede lograr una mayor comprensión de la enfermedad y finalmente aplicar estos conocimientos en la vida diaria logrando la consolidación de hábitos alimentarios saludables adaptados a la condición celiaca que permitan mejorar su estado de salud.

PALABRAS CLAVE: Enfermedad Celiaca - Game-based learning - videojuegos educativos - serious games - aprendizaje basado en juegos - comunicación.

\section{ABSTRACT:}

This study proposes the design of a video game based on the game-based learning methodology as a means of communication to facilitate the formation of healthy eating habits in children with a diagnosis of Celiac Disease (CD). The need arises from what is observed in the professional practice in the nutrition consultation, where mothers of celiac patients express as their main concern the fact that the child, exposed to various social and academic activities, in which they are not necessarily with their company, be able to comply with the gluten-free diet, the only treatment for CD. The research is based on the concept and clinical manifestations of $C D$, the use of games as a teaching resource (game-based learning), and the theoretical vision of the constructivist paradigm of learning. Due to its characteristics, this research is congruent with the Feasible Project, at a descriptive level and is supported by documentary research using the analysis technique and the documentary record as an instrument for obtaining information. It was developed in two phases, a preparatory phase where the necessary documentary information was obtained to fulfill the proposed objectives and a design phase that consisted of the development of the instructional components of the video game and the approach to a prototype. The video game designed is then an educational game (serious game) called El Mundo de Celia, which consists of a world in the form of a board, composed of three levels, where the participant must overcome nine challenges in total, and by practicing the game, you can achieve a greater understanding of the disease and finally apply this knowledge in daily life, achieving the consolidation of healthy eating habits adapted to the celiac condition that allow you to improve your health.

KEY WORDS: Celiac disease - Game-based learning - educational video games serious games - game-based learning - communication. 


\section{VIDEOGAME A PARTIR DA METODOLOGIA GAME-BASED LEARNING COMO FORMA DE COMUNICAÇÃO PARA A FORMAÇÃO DE HÁBITOS ALIMENTARES PARA CRIANÇAS COM DOENÇA CELÍACA}

\section{RESUMO}

Neste estudo se propõe a criação de um videogame baseado na metodologia gamebased learning como forma de comunicação para facilitar a formação de hábitos alimentares saudáveis em crianças com diagnóstico de doença Celíaca (DC). A necessidade surge do observado na prática profissional na consulta de nutrição, onde mães de pacientes celíacos manifestam como preocupação principal o fato de que a criança, exposta a diversas atividades sociais e acadêmicas, nas quais não necessariamente se encontra na sua companhia, seja capaz de cumprir a dieta livre de glúten, único tratamento para a DC. A pesquisa se fundamenta no conceito e manifestações clínicas da DC, o uso do jogo como recurso para o ensino (gamebased learning), e a visão teórica do paradigma construtivista do aprendizado. Pelas suas caraterísticas, esta pesquisa resulta congruente com o Projeto viável, em um nível descritivo e se apoia na pesquisa documental usando a técnica de análise e registro documental como instrumento para a obtenção de informação. Foi feita em duas fases, uma fase preparatória onde se obteve a informação documental necessária para o cumprimento dos objetivos propostos e uma fase de design que consistiu no desenvolvimento dos componentes instrucionais do videogame e a aproximação para um protótipo. O videogame projetado é então educativo (serious game) chamado El Mundo de Celia, que está composto por um mundo em forma de tabuleiro, composto por três níveis, onde o participante deve superar nove desafios no total, e mediante a prática do jogo, pode se obter uma maior compreensão da doença e finalmente aplicar estes conhecimentos na vida diária alcançando a consolidação de hábitos alimentares saudáveis adaptados a condição celíaca que permitam melhorar seu estado de saúde.

PALAVRAS CHAVE: Doença Celíaca - Game-based learning - videogames educativos - serious games - aprendizado baseado em jogos - comunicação.

\section{Cómo citar el artículo:}

Santos Rivera, J. M. y Navas Piñate, E. E. (2020). Juego de vídeo a partir de la metodología game-based learning como medio de comunicación para la formación de hábitos alimentarios en niños con enfermedad celíaca. [Video game based on the game-based learning methodology as a means of communication for the formation of eating habits in children with celiac disease]. Revista de Comunicación de la SEECI, 53, 83-101. doi: https://doi.org/10.15198/seeci.2020.53.83-102

Recuperado de http://www.seeci.net/revista/index.php/seeci/article/view/661 
Santos Rivera, J. M. y Navas Piñate, E. E. Juego de vídeo a partir de la metodología gamebased learning como medio de comunicación para la formación de hábitos alimentarios en niños con enfermedad celíaca

\section{INTRODUCCIÓN}

La Enfermedad Celíaca (EC), conocida también como celiaquía, es una condición en la cual el sistema inmunológico del individuo identifica como antígeno (una sustancia ajena al organismo) al gluten, específicamente a la gliadina que se encuentra en el trigo y otras proteínas afines presentes en cereales como: cebada, centeno y en menor concentración en la avena; desencadenando una reacción inmunológica en personas con una predisposición genética, provocando una serie de síntomas que parten de un daño en las vellosidades intestinales que se traduce en un defecto en la absorción de nutrientes en el tracto digestivo (Fabiano, F., Lista, D., Torres, J., y Urquiola, A., 2013, p. 204, y Ministerio de Salud de Argentina, 2014, p. $5)$.

El único tratamiento efectivo para esta condición consiste en retirar este "antígeno" de la dieta, es decir, el paciente debe llevar a lo largo de su vida una alimentación libre de gluten, mientras más temprano se comience el plan de alimentación adecuado, más rápida será la recuperación de la mucosa intestinal y la desaparición de los síntomas.

En la consulta nutricional, una de las preocupaciones más manifestadas por las madres de estos niños es la adhesión al tratamiento. Cuando el niño es pequeño, su alimentación depende de la madre o del adulto que se encarga de su crianza, pero a medida que va creciendo se va haciendo más independiente y su círculo social se va ampliando. Este cambio, en la mayoría de los casos incluye la posibilidad de que el niño se exponga, estando sin el adulto, a la presencia de alimentos que le puedan ocasionar daño, y supone entonces de su parte un entendimiento profundo de su condición y del tratamiento, que le permita desarrollar la voluntad necesaria para abstenerse de consumir estos alimentos que desencadenan nuevamente la sintomatología. A esto, se suma el hecho de que en la literatura se registra una adhesión a la dieta libre de gluten que vagamente alcanza el $45 \%$ de los casos de niños (Amigo citado en Aznar, 2009, Bravo y Paz, 2011), donde aproximadamente el $70 \%$ de los casos encuentra la dieta difícil de seguir y más de un $40 \%$ no sabe que comer. (Bravo y Paz, 2011)

Actualmente, existe una gran cantidad de información disponible en la Internet acerca de esta condición. Se han utilizado distintos recursos como cuentos infantiles sobre la enfermedad y algunos recursos lúdicos. Sin embargo, este tipo de recursos se han hecho pensando sobre todo en ese niño que aún depende de su madre.

Desde hace unos años, la tecnología ha venido ganando terreno como facilitador del proceso de enseñanza y aprendizaje y mediador comunicacional, y en esa gama de herramientas tecnológicas de las cuales se dispone actualmente se encuentran los juegos de video. Jugar es una actividad natural del ser humano y por medio del juego se desarrollan habilidades sociales, cognitivas y se logra motivar al aprendizaje (Perrota et al; Kenny y MacDaniel; Kirriemuir y McFarlane; Higgins et al, citados en Contreras, 2016) 
Santos Rivera, J. M. y Navas Piñate, E. E. Juego de vídeo a partir de la metodología gamebased learning como medio de comunicación para la formación de hábitos alimentarios en niños con enfermedad celíaca

Planteado esto surge la interrogante ¿de qué manera puede un juego de vídeo facilitar la enseñanza de contenidos asociados con la enfermedad celíaca propiciando, en el niño, la formación de hábitos alimentarios adecuados a esa condición?

\section{OBJETIVOS.}

A partir de la situación planteada, se establece que el objetivo general que orientó este estudio fue diseñar un juego de video a partir de la metodología game-based learning para facilitar la formación de hábitos alimentarios adecuados en niños con diagnóstico de Enfermedad Celíaca. Para el logro del objetivo general, se concibieron los siguientes objetivos específicos:

- Definir los aspectos de la metodología game-based learning a ser desarrollados en el juego de video.

- Caracterizar el perfil nutricional del niño de 7 a 10 años, con diagnóstico de enfermedad celíaca.

- Establecer el cuerpo de recomendaciones acordes al perfil nutricional de un niño de 7 a 10 años, con diagnóstico de enfermedad celíaca.

- Desarrollar los componentes instruccionales que conforman el juego de video.

- Esbozar el diseño del juego de vídeo partiendo de los componentes instruccionales desarrollados.

\section{METODOLOGÍA.}

Tal como lo especifica la Universidad Pedagógica Experimental Libertador (UPEL) (2016):

El Proyecto Factible consiste en la investigación, elaboración y desarrollo de una propuesta de un modelo operativo viable para solucionar problemas, requerimientos o necesidades de organizaciones o grupos sociales; puede referirse a la formulación de políticas, programas, tecnologías, métodos o procesos. (p. 21).

De acuerdo con esa definición, esta investigación responde a las características mencionadas ya que pretende dar solución a la necesidad detectada mediante el diseño de un juego de video como medio comunicacional a partir de la metodología game-based learning para facilitar la formación de hábitos alimentarios adecuados en niños de 7 a 10 años con diagnóstico de Enfermedad Celíaca. Asimismo, se ubica en un nivel descriptivo y se apoya en la investigación documental utilizando la técnica de análisis y el registro documental como instrumento para la obtención de información.

En aras del cumplimiento del objetivo general de esta investigación se podría dividir el procedimiento llevado a cabo en dos fases:

- Fase preparatoria: comprendida por la contextualización del tema de estudio y la revisión documental que permitió la consolidación del marco teórico donde se establecen los contenidos, así como las estrategias y métodos a utilizar en el diseño del videojuego propuesto desde el punto de vista instruccional, 
Santos Rivera, J. M. y Navas Piñate, E. E. Juego de vídeo a partir de la metodología gamebased learning como medio de comunicación para la formación de hábitos alimentarios en niños con enfermedad celíaca

tomando en cuenta los aspectos relacionados con el enfoque game-based learning y los componentes que deben integrar el juego para poder ser catalogado como «videojuego».

El análisis de la información recolectada permitió establecer una aproximación al perfil nutricional del niño con EC, así como un cuerpo de recomendaciones para la alimentación saludable de este tipo de paciente. Asimismo, permitió esbozar las actividades que estarían incluidas para el cumplimiento de los objetivos de aprendizaje del videojuego.

- Fase de diseño: comprendida por el desarrollo del diseño instruccional propuesto para el juego de video donde se contempla su propósito así como los objetivos de aprendizaje que se espera que los usuarios alcancen, las estrategias y métodos a aplicar, y por último, las actividades que deberán superar a fin de alcanzar los objetivos propuestos. En esta fase se seleccionaron, de igual forma, algunos de los componentes gráficos que se sugieren para el juego, así como un lenguaje de programación a fin de lograr una aproximación a un prototipo funcional que permitiera al lector visualizar, al menos parcialmente, el producto final materializado.

Para desarrollar el diseño instruccional se realizó una adaptación del modelo de diseño propuesto por el Prof. Joel Aguilar, profesor de la Universidad Metropolitana y la Universidad Simón Bolívar (Caracas, Venezuela) con amplia trayectoria en el área del diseño instruccional. De acuerdo con este modelo, el diseño instruccional está integrado por una serie de componentes preliminares (justificación, necesidad, contexto y propósito), esenciales (expresiones de logro, estructura del contenido y estrategias de evaluación) y específicos (estrategias, métodos, técnicas, recursos y criterios de ejecución), todos estos reformulables, modificables e incluso fusionables; esta plasticidad del modelo y su versatilidad fueron determinantes en la selección.

En esta fase también se realizó el esbozo del juego partiendo de sus componentes instruccionales y la programación de una de las actividades utilizando el lenguaje Scratch. Se seleccionó para ello la segunda actividad o reto que estaría contenida en el Nivel 2 del juego.

Los aspectos relacionados con el desarrollo de la propuesta, enfatizando el diseño instruccional donde se encuentran el propósito, los objetivos de aprendizaje, los contenidos, así como las actividades diseñadas para el videojuego propuesto y finalmente la aproximación al prototipo del juego se describen en el epígrafe a continuación.

\section{RESULTADOS.}

Se presenta a continuación el diseño propuesto desde el punto de vista instruccional para un juego de vídeo que funcione como aliado en la formación de hábitos de alimentación adecuados para niños con diagnóstico de Enfermedad 
Santos Rivera, J. M. y Navas Piñate, E. E. Juego de vídeo a partir de la metodología gamebased learning como medio de comunicación para la formación de hábitos alimentarios en niños con enfermedad celíaca

Celiaca. Así mismo, se hace referencia a los aspectos relacionados con una aproximación al prototipo del juego partiendo de sus componentes instruccionales.

\subsection{DISEÑO INSTRUCCIONAL DEL JUEGO DE VIDEO}

\subsubsection{Justificación}

La Enfermedad Celiaca (EC) es una enfermedad autoinmune que se desarrolla en personas genéticamente susceptibles en la cual el sistema inmunológico del individuo identifica como antígeno a un grupo de proteínas llamadas prolaminas, a las cuales pertenece el gluten que se encuentra en diversos cereales como son: trigo, cebada, centeno y en menor medida en la avena. (Fabiano, Lista, Torres y Urquiola, 2013, p. 204, y Ministerio de Salud de Argentina, 2014, p. 5)

El problema de esta reacción es que la acción del sistema inmunológico se traduce en un daño a la mucosa gástrica del paciente que progresivamente impide la absorción de nutrientes, hecho que se manifiesta con síntomas gastrointestinales (en los casos de la forma clásica de la enfermedad) y el compromiso del estado nutricional con retraso en el crecimiento y desarrollo del paciente. (Polanco y Ribes, 2010, Fabiano, Lista, Torres y Urquiola, 2013, y Ministerio de Salud de Argentina, 2014)

De manera que, el único tratamiento posible para revertir esta condición es eliminar de la dieta todos aquellos alimentos y productos en general (como medicamentos) que contengan prolaminas. No existe un tratamiento farmacológico. (Polanco y Ribes, 2010, y Fabiano, Lista, Torres y Urquiola, 2013) Por esta razón, es de vital importancia para el paciente celiaco desarrollar un profundo entendimiento de su enfermedad y del tratamiento a fin de lograr la recuperación.

En este sentido, contar con una herramienta que permita, en un lenguaje sencillo, proveer al paciente de información acerca de la enfermedad y su tratamiento resulta de gran utilidad.

\subsubsection{Necesidad instruccional}

Cuando llegan casos de niños diagnosticados con Enfermedad Celiaca a la consulta de nutrición una de las preocupaciones manifestadas por las madres con mayor frecuencia es la incertidumbre acerca de si el niño a medida que va creciendo podrá cumplir la dieta en ausencia de la madre, tomando en cuenta la presión social a la que puede exponerse en ocasiones inherentes a sus actividades académicas y sociales. Este hecho, depende en gran medida de que para ese momento el paciente haya comprendido de la forma más profunda posible de qué se trata su enfermedad, cómo se cumple con el tratamiento y cuáles son las consecuencias de su incumplimiento. Por lo tanto, desde el momento del diagnóstico, el niño debe ser provisto de información suficiente acerca de su condición. 
Santos Rivera, J. M. y Navas Piñate, E. E. Juego de vídeo a partir de la metodología gamebased learning como medio de comunicación para la formación de hábitos alimentarios en niños con enfermedad celíaca

Tratándose de niños es muy importante que la información sea presentada de acuerdo con el desarrollo cognitivo que haya alcanzado en concordancia con su edad. Para esto, valerse de herramientas tecnológicas, es una decisión inteligente ya que permiten transmitir la información adaptando el lenguaje y estrategias utilizadas para ello, toda vez que motiva al aprendiz, en este caso al paciente, a querer aprender.

\subsubsection{Contexto de la instrucción.}

Se trata de un videojuego educativo dirigido a niños de 7 a 10 años con diagnóstico de Enfermedad Celiaca que tengan conocimientos previos de su condición.

\subsubsection{Propósito.}

Compartir un compendio de conocimientos que le permitan al participante el reconocimiento de las características de la enfermedad celiaca y su tratamiento, y a partir de ello, proponerse un plan de hábitos saludables adaptados a su condición expresados por medio del complimiento de la dieta libre de gluten con sustento en criterios básicos adquiridos a medida que supera los distintos retos del juego.

\subsubsection{Objetivo General.}

Reconocer las características de la enfermedad celiaca y su tratamiento, a medida que se superan los distintos retos presentados en el juego, con el fin de aplicar estos conocimientos en la vida diaria logrando la consolidación de hábitos alimentarios saludables adaptados a la condición celiaca que permitan mejorar el estado de salud del participante.

\subsubsection{Objetivos de aprendizaje.}

- Desarrollar una plataforma de conceptos relacionados al área de conocimiento.

- Aplicar el marco conceptual y teórico para reconocer las características de la enfermedad celiaca y su tratamiento.

- Caracterizar los componentes de una dieta saludable adaptada a la condición celiaca.

\subsubsection{Contenidos seleccionados.}

Debido a que la EC se trata de una condición en la cual el sistema inmunológico del paciente reacciona ante la presencia del gluten contenido en los alimentos que consume y esta reacción causa un daño en la mucosa gástrica que va deteriorando su salud, en ausencia de tratamiento farmacológico, el único tratamiento posible y efectivo es precisamente un cambio en la alimentación que consiste en la remoción de todo aquel alimento que contenga gluten y cualquier otro nutriente que pueda exacerbar o hacer aparecer los síntomas, dependiendo de la gravedad del daño. 
Santos Rivera, J. M. y Navas Piñate, E. E. Juego de vídeo a partir de la metodología gamebased learning como medio de comunicación para la formación de hábitos alimentarios en niños con enfermedad celíaca

Por esta razón los contenidos seleccionados para ser abordados mediante el uso del juego se concentran en información acerca de lo que es la enfermedad, qué la causa y cómo puede mejorarse. Estos contenidos se presentan en la tabla a continuación (ver Tabla 1)

Tabla 1. Contenidos sugeridos para el juego de vídeo.

\begin{tabular}{cc}
\hline Nivel & Contenido \\
\hline Nivel 1 & $\begin{array}{c}\text { 1. Definición de Enfermedad Celiaca. } \\
\text { 2. Clasificación de la Enfermedad Celiaca. } \\
\text { Nivel } 2\end{array}$ \\
3. Síntomas más comunes de la Enfermedad Celiaca. \\
4. El gluten como desencadenante de los síntomas. \\
5. Alimentos que contienen gluten. \\
6. Reconocimiento de alimentos comerciales con gluten. \\
7. Qué es la dieta sin gluten o dieta libre de gluten. \\
8. Aprende qué alimentos son permitidos, riesgosos y no permitidos \\
9. Metas del tratamiento y nociones acerca de la alimentación saludable \\
adaptada a la condición celiaca.
\end{tabular}

Fuente: Elaboración propia

\subsubsection{Estrategias de evaluación.}

Debido a que se trata del diseño de un juego de video los retos que supera el jugador representan una evaluación de tipo formativa siendo que cada reto superado arroja un mensaje, distinto en cada caso, de refuerzo del conocimiento.

Asimismo, la superación de todos los retos en conjunto arrojará una puntuación que le permite al jugador pasar al siguiente nivel siendo la globalidad de las actividades una evaluación sumativa. En la Tabla 2 se pueden observar las actividades que los participantes deberán ejecutar.

Tabla 2. Actividades de evaluación que conformarán los retos del juego

\begin{tabular}{|c|c|c|c|c|}
\hline Nivel & Actividad & Descripción & $\begin{array}{l}\text { Contenido } \\
\text { abordado }\end{array}$ & Recompensa \\
\hline 1 & $\begin{array}{l}\text { Atrapa las palabras que } \\
\text { se relacionan con la } \\
\text { definición y tipos de } \\
\text { enfermedad celiaca. }\end{array}$ & $\begin{array}{l}\text { En esta actividad el } \\
\text { participante debe lograr } \\
\text { que el personaje principal } \\
\text { recolecte en una caja que } \\
\text { aparece en pantalla las } \\
\text { palabras que se } \\
\text { relacionan con la } \\
\text { definición de EC } \\
\text { previamente dada y evite } \\
\text { aquellas que no están } \\
\text { relacionadas. }\end{array}$ & 1,2 & Puntaje \\
\hline
\end{tabular}


Santos Rivera, J. M. y Navas Piñate, E. E. Juego de vídeo a partir de la metodología gamebased learning como medio de comunicación para la formación de hábitos alimentarios en niños con enfermedad celíaca

Salta los cubos identificados con imágenes que reflejan síntomas de la EC.

Escoge las moléculas identificadas con la palabra gluten y sácalas para que no lleguen al intestino.

Ayuda a Celia a formar un desayuno con alimentos que no contengan gluten.

Selecciona alimentos identificados como libres de gluten para ayudar a Celia a llenar su carrito de mercado.

Recuerda evitar llegar al final del recorrido con algún alimento no deseado.

3 Deja en el baúl las palabras o imágenes relacionadas con lo que es la Dieta Libre de Gluten (DLG).
En esta actividad el participante debe lograr esquivar saltando aquellos cubos que aparecen en el camino identificados con imágenes que reflejan síntomas de la EC.

Se muestra una representación gráfica del estómago recibiendo distintos nutrientes y moléculas conectado con el intestino. El participante debe tomar aquellas identificadas como "gluten" y sacarlas para que no lleguen al intestino.

Se muestra en pantalla una nevera llena de alimentos. El participante debe seleccionar aquellos que no contienen gluten para armar su desayuno. Se muestra en pantalla una escena donde el avatar recorre un supermercado. El participante debe seleccionar y meter en el carrito alimentos etiquetados como libres de gluten. De igual forma, en este reto aparece un personaje que, sin que el personaje principal lo note, introduce alimentos no adecuados en el carrito. El participante debe estar atento y sacarlos antes de llegar al final de recorrido.

Para esta actividad aparece en pantalla un baúl lleno de palabras e imágenes. El participante debe lograr que avatar principal extraiga del baúl todas aquellas palabras que no están relacionadas con lo que es la DLG y dejar solamente aquellas que si lo están. 4 Puntaje

3

Puntaje

5

Puntaje

6

Puntaje

7

Puntaje 
Santos Rivera, J. M. y Navas Piñate, E. E. Juego de vídeo a partir de la metodología gamebased learning como medio de comunicación para la formación de hábitos alimentarios en niños con enfermedad celíaca

Organiza los alimentos
ubicándolos en la
canasta a la cual
pertenecen.

Conduce cada una de las pistas para alcanzar cada una de las metas del tratamiento de la enfermedad celiaca. Recuerda esquivar aquellos alimentos que son un obstáculo para que tengas hábitos que te mantengan saludable.

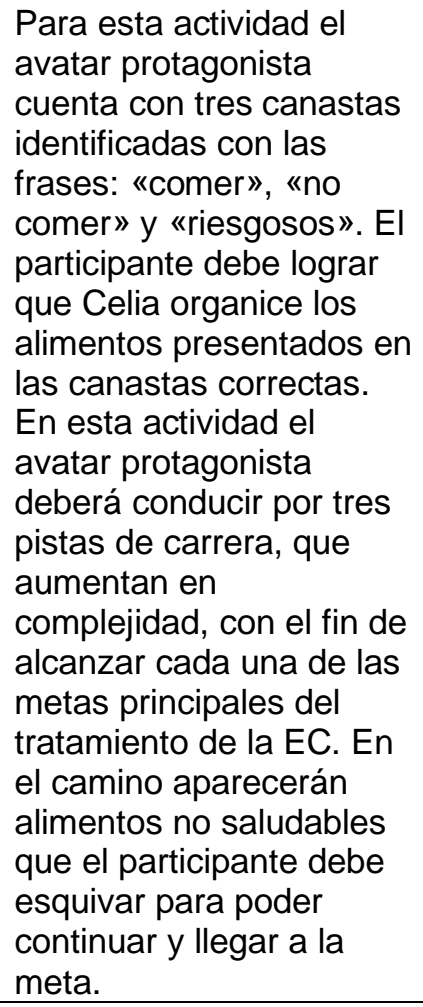

Fuente: Elaboración propia

\subsubsection{Estrategias y métodos de aprendizaje.}

La estrategia utilizada es el Game-based learning o aprendizaje basado en juegos que consiste en la integración de juegos reales en el proceso de aprendizaje usualmente para enseñar habilidades específicas o alcanzar un objetivo específico (Israel, 2017, p. 3). El GBL se apoya en el uso del juego como un mediador para la mejor comprensión de contenidos, que se encuentran inmersos en el juego, o el desarrollo de habilidades específicas.

Los factores que son estimulados en el participante por el videojuego y que hacen el proceso de aprendizaje efectivo son: motivación, concentración, atención y emoción (McFarlane, Sparrowhawk y Heald; Malone y Lepper; y Keller y Kopp citados en Padilla, González, Gutiérrez, Cabrera y Paderewski, 2009)

Por medio de las pantallas del juego se utilizarán escenas demostrativas que permitirán al participante comprender los conceptos presentados mediante la exposición de situaciones y ejemplos relacionados con la enfermedad.

\subsection{Esbozo del juego partiendo de los componentes instruccionales}

Se sugiere como nombre para el videojuego «El Mundo de Celia», utilizar «Celia» como nombre para el personaje que desarrolla las acciones hace alusión al término «Celiaquía» que es la condición que se aborda con el juego. Asimismo, los nombres 
Santos Rivera, J. M. y Navas Piñate, E. E. Juego de vídeo a partir de la metodología gamebased learning como medio de comunicación para la formación de hábitos alimentarios en niños con enfermedad celíaca

del resto de los personajes se asocian tanto con la condición como con los especialistas involucrados en su tratamiento.

El juego se compone de tres personajes:

- Celia: es el personaje central con el cual el participante juega, se comunica y desarrolla la mayoría de las acciones.

- Dr. Glutenfree: es un narrador que aparece al inicio del juego y durante el desarrollo de los retos que se relacionan con la definición y sintomatología de la enfermedad, para dar retroalimentación y asignar puntajes.

- Nutrigirl: es un narrador que aparece al inicio del juego y durante el desarrollo de los retos que se relacionan directamente con la alimentación, para dar retroalimentación y asignar puntajes.

Partiendo de la propuesta de Padilla, González, Gutiérrez, Cabrera y Paderewski (2008) para el diseño de este juego educativo se sugiere una estructura acorde con la estructuración de los contenidos seleccionados. Así, se parte del concepto de «Mundo» que corresponde a la interface, el lugar donde se desarrollan las acciones. En el mundo se enseña una unidad didáctica. A su vez, el Mundo se compone de «Niveles» en los cuales se abarcan los contenidos conceptuales y procedimentales para la asimilación de los conceptos. Finalmente, cada Nivel se compone de «Retos» o pruebas a realizar que corresponden a las actividades del momento de instrucción. En la Tabla 3 se observa un ejemplo de esta equivalencia.

Tabla 3. Ejemplo de correspondencia de estructura del juego con elementos del proceso de enseñanza

\begin{tabular}{|c|c|c|}
\hline Aspecto Lúdico & $\begin{array}{c}\text { Factor en el proceso de } \\
\text { Enseñanza }\end{array}$ & Ejemplo \\
\hline Mundo & Unidad Didáctica & Enfermedad Celiaca \\
\hline Nivel & $\begin{array}{l}\text { Contenido conceptual y/o } \\
\text { procedimental }\end{array}$ & $\begin{array}{l}\text { Dieta libre de } \\
\text { gluten/Reconocimiento de alimentos } \\
\text { libres de gluten. }\end{array}$ \\
\hline Reto & Actividad & $\begin{array}{l}\text { Logra que Celia coma solo los } \\
\text { alimentos que no contienen qluten. }\end{array}$ \\
\hline
\end{tabular}

Fuente: Elaboración propia

En el caso particular del «Mundo de Celia», el Mundo, se compone de tres Niveles. En el nivel 1 se abarcan los contenidos correspondientes al concepto de Enfermedad Celiaca, su definición y algunos atributos como los síntomas y la clasificación de la enfermedad. En el nivel 2 se abarca el concepto de gluten como desencadenante de la EC, y atributos de este concepto como los alimentos que contienen gluten y el reconocimiento de alimentos comerciales que también lo contienen mediante el uso de la simbología del etiquetado. Finalmente, en el nivel 3 se abarcan contenidos conceptuales como «Dieta libre de gluten» por medio de su definición, así como contenidos procedimentales como el reconocimiento de alimentos permitidos, no 
Santos Rivera, J. M. y Navas Piñate, E. E. Juego de vídeo a partir de la metodología gamebased learning como medio de comunicación para la formación de hábitos alimentarios en niños con enfermedad celíaca

permitidos y riesgosos, y alimentos que entran dentro de una alimentación saludable adaptada al paciente celiaco.

El objetivo del juego es lograr que Celia, el avatar protagonista del juego, se mantenga durante todas las partidas en un excelente estado de salud mediante la superación del cada reto. Al inicio del juego, Celia dispone de tres vidas que puede perder y volver a recuperar. Las vidas se pierden cuando el reto no es superado con la cantidad de puntos requerida y se recuperan con una cantidad de puntos específica obtenidos mediante la superación de los retos. Las definiciones de los conceptos son dadas por Celia y los narradores en la introducción del juego y reforzadas por los narradores durante el desarrollo de cada nivel.

Es muy importante que la programación de las actividades parta de un banco de elementos. Por ejemplo, si la actividad consiste en recoger palabras asociadas con un concepto, en la programación se debe contar con un banco de palabras tanto asociadas como no asociadas, de cantidad suficiente, de manera que en cada partida aparezcan palabras distintas aleatoriamente, evitando de esta forma la activación del conocimiento memorístico.

El juego inicia con una pantalla principal donde el participante puede seleccionar si desea leer o escuchar la historia de Celia. Una vez efectuada la selección, en la segunda pantalla, mediante el uso de globos de texto interactivos, gráficos (que están presentes independientemente de la opción seleccionada) y sonido (que aparece solamente cuando el participante selecciona la opción "escuchar" o cuando hace clic en el globo de texto) Celia explica quién es y que ha sido diagnosticada con la enfermedad. La pantalla siguiente presenta una serie de fichas interactivas, e ilustraciones, con información acerca de lo que es y lo que no es la Enfermedad Celiaca y las implicaciones que tiene para el paciente, explicadas por el «Dr. Glutenfree» y «Nutrigirl». Seguidamente se muestra una pantalla donde aparecen Celia, el Dr. Glutenfree y Nutrigirl con una serie de alimentos naturales e industriales, donde se explica cuáles contienen gluten, cuáles podrían ser un riesgo y cuáles no contienen gluten. Por último, utilizando globos de texto interactivos, Celia realiza un recuento, demostrando que ha comenzado a entender cómo funciona la enfermedad.

Posteriormente aparece una pantalla donde se observa el mundo de Celia a manera de tablero con los 3 niveles que lo conforman y que el participante deberá superar para llegar al final de juego. Los niveles se encontrarán identificados con iconografía alusiva a los contenidos y conectados con un camino en el cual el participante avanza cuando supera cada nivel.

El primer nivel del juego se compone de dos retos. Por su parte, el segundo y tercer nivel se componen de tres retos cada uno. Para avanzar al siguiente, el participante debe superar el reto previo.

Al inicio de esta actividad el personaje del Dr. Glutenfree aparece para dar las instrucciones del reto y de la misma manera continuará apareciendo durante todo su desarrollo para dar mensajes de refuerzo tanto si el paciente acierta como si 
Santos Rivera, J. M. y Navas Piñate, E. E. Juego de vídeo a partir de la metodología gamebased learning como medio de comunicación para la formación de hábitos alimentarios en niños con enfermedad celíaca

comente algún error. Esta modalidad de retroalimentación es constante en cada una de las actividades.

Las palabras irán apareciendo en caída, unas relacionadas y otras no, de manera que el niño debe seleccionar solamente las correctas. Es muy importante destacar nuevamente que el juego debe partir de un banco de alimentos, palabras, gráficos, imágenes; que permita que cada partida sea distinta a la anterior lo que significa que las palabras correctas e incorrectas no siempre serán las mismas a fin de prevenir el aprendizaje memorístico.

Al inicio, como en cada actividad, uno de los personajes da las instrucciones y acompaña el desarrollo del juego, en el caso particular de esta actividad, este rol lo cumple Celia, el avatar principal del juego.

\subsection{Aproximación al prototipo}

Con el fin de poder evidenciar la factibilidad de la propuesta se planteó la aproximación a un prototipo funcional mediante la codificación en lenguaje de programación de una de las actividades propuestas para el juego en su diseño instruccional.

\subsubsection{Selección de la herramienta para la aproximación al prototipo.}

Para la aproximación al prototipo del videojuego se revisaron distintas herramientas de programación. De esta revisión, finalmente se seleccionó Scratch como herramienta para este propósito.

Scratch es un lenguaje de programación visual basado en bloques "a diferencia de otros lenguajes de programación como python, php, entre otros, que necesitan ser escrito en símbolos o códigos, en Scratch solo debemos arrastrar y unir bloques." (Academy Pop, 2019) De esta manera se expresan las ideas para desarrollar historias interactivas, juegos y animaciones. Scratch también funciona como una "comunidad en línea donde los niños pueden programar y compartir medios interactivos...con gente de todo el mundo" (Scratch, 2019).

Las principales razones por las cuales se seleccionó esta herramienta luego de haberla revisado fueron:

- Es gratuita.

- Es sencilla de utilizar.

- Permite la inclusión de todos los elementos que forman parte de un videojuego.

- Permite el desarrollo de las actividades incluidas en el diseño instruccional del videojuego propuesto en esta investigación.

- Es un lenguaje sencillamente modificable de manera que posibilita la actualización y mejoramiento periódico del juego. 
Para esta primera fase se desarrolló una sola actividad de las diseñadas para el juego. La actividad corresponde al segundo reto del Nivel 2 del juego, con la cual se abordaría el quinto contenido. Es muy importante recordar que para el momento de esta actividad el participante ha recibido una cantidad de información tanto en la introducción, como en actividades previas, que le facilita la superación de este reto.

En este caso, como narrador se utiliza una versión de pie del avatar que representa a Nutrigirl, quien se encarga de dar las instrucciones del juego, al inicio de la partida, de forma verbal y escrita. La comunicación ocurre por medio de globos de texto con parte de las instrucciones que recibe el participante.

Scratch posee una sección llamada «Estudio» donde el programador puede agrupar distintos proyectos relacionados entre sí. De manera que, con esta herramienta, en primera instancia se podrían programar las diferentes actividades y contenidos de todo el juego, que reposarían en un mismo Estudio titulado de la misma forma que el videojuego. Sin embargo, la forma como está diseñado desde el punto de vista instruccional, hace que cobre importancia tener una secuencia en la resolución de las distintas actividades o retos. Por este motivo, es importante el diseño del juego como un solo bloque, donde es necesario superar el primer nivel para pasar al segundo y así sucesivamente. En este sentido, mediante la revisión de distintos foros de Scratch se encontró que algunos programadores sugerían, para este propósito, intentar la codificación de un software intermediario, en algún otro lenguaje de programación (como Python, por ejemplo), que permita la conexión de Scratch con una base de datos, de forma que el avance del jugador pueda guardarse; esto conformaría una fase siguiente a la implementación de esta investigación. (Technoguyx, 2009 y Monrroy, 2009).

\section{CONCLUSIONES.}

La observación directa de pacientes en la consulta es una situación que definitivamente sensibiliza. Cada caso es particular, distinto. Sin embargo, cuando se trata de pacientes con enfermedades crónicas, que por su naturaleza, afectan todos los aspectos de la vida de ese paciente, la sensibilización ocurre de manera más acelerada.

Tomando en cuenta esto, se seleccionó la rama de la educación en salud para vincularla con la tecnología, específicamente con las TIC, en la búsqueda de un recurso que permitiera, de alguna forma, mejorar la calidad de vida de este tipo de paciente, y en este caso, pacientes infantiles diagnosticados con Enfermedad Celiaca.

Por esta razón el objetivo general de esta investigación fue «Diseñar un juego de video a partir de la metodología game-based learning para facilitar la formación de hábitos alimentarios adecuados en niños con diagnóstico de Enfermedad Celíaca.» Con el propósito de lograr este objetivo, se plantearon cinco objetivos específicos alcanzados partiendo de la revisión documental y el análisis de la información revisada. 
En primer lugar, se definieron los aspectos del enfoque game-based learning a ser desarrollados en el juego de video, entendiendo que se caracteriza principalmente por el uso de juegos reales como aliados para la enseñanza y el aprendizaje, bien sea que ya existieran y hayan sido adaptados para este fin, o que desde su concepción hayan sido creados partiendo de objetivos de aprendizaje definidos (juegos serios) sin perder sus características lúdicas en ningún caso. De manera que, los aspectos más relevantes del GBL para ser desarrollados en el videojuego fueron precisamente el uso del juego como recurso de enseñanza y mediador del proceso de aprendizaje y la conservación del aspecto lúdico del juego aun cuando, en este caso particular, fuera concebido partiendo de objetivos de aprendizaje previamente definidos.

El segundo objetivo planteado fue «Caracterizar el perfil nutricional del niño de 7 a 10 años, con diagnóstico de Enfermedad Celiaca» para lograrlo se realizó una investigación de distintas fuentes bibliográficas y estudios clínicos. Entendiendo que la mayoría de los estudios no se refiere a grupos de edad específicos, sino que categorizan en adultos y niños en general, la información revisada fue suficiente para comprender que el perfil del niño diagnosticado con esta enfermedad antes del tratamiento se asocia con algunos de los síntomas propios de la condición como son: bajo peso, con hipotrofia muscular y retraso en el crecimiento, así como manifestaciones producto de la deficiencia específica de algunos nutrientes, como por ejemplo anemia ferropénica.

En contraste, en el niño tratado, generalmente se observará un peso adecuado y en algunos casos sobrepeso, la activación del proceso de crecimiento, y aunque algunas deficiencias de nutrientes persistirán mientras ser revierte el daño de la mucosa, en líneas generales, logrando una alimentación equilibrada, su condición nutricional será adecuada.

Como tercer objetivo se planteó el establecimiento de un cuerpo de recomendaciones nutricionales para el niño con diagnóstico de EC. Esto fue posible partiendo de las «Recomendaciones de Energía y Nutrientes para la población venezolana», y tomando en cuenta las posibles deficiencias de nutrientes que podrían presentarse.

Como cuarto objetivo específico se planteó el desarrollo de los componentes instruccionales del juego. En este sentido es importante resaltar que en el diseño instruccional logrado se estableció como propósito para este videojuego compartir un compendio de conocimientos relacionados con la enfermedad celiaca y su tratamiento para que el usuario pueda reconocer las características de la enfermedad y a partir de ello, proponerse el complimiento de la dieta libre de gluten desde un entendimiento más amplio de la condición. Con un propósito claro, se espera que los usuarios del juego, a medida que superan los distintos retos presentados, puedan lograr una mayor comprensión de la enfermedad y finalmente aplicar estos conocimientos en la vida diaria logrando la consolidación de hábitos alimentarios saludables adaptados a la condición celiaca que permitan mejorar su estado de salud. 
Santos Rivera, J. M. y Navas Piñate, E. E. Juego de vídeo a partir de la metodología gamebased learning como medio de comunicación para la formación de hábitos alimentarios en niños con enfermedad celíaca

Se diseñó entonces un videojuego, fundamentado en el paradigma constructivista del aprendizaje, llamado «El Mundo de Celia», cuyo tablero conforma un mundo (que vendría siendo la unidad didáctica) compuesto por tres niveles (que contienen los conceptos y conocimientos procedimentales) y cada nivel compuesto por retos (confeccionados a partir de las actividades establecidas en el diseño). El primer nivel se compone de dos retos que abarcan contenidos conceptuales y los niveles 2 y 3 contienen cada uno tres retos a superar que contemplan mayormente contenidos procedimentales.

Finalmente, el quinto objetivo consistió en un esbozo del juego partiendo de los componentes instruccionales desarrollados, mismo que fue logrado mediante la diagramación y graficación de algunas de las pantallas del juego, así como con la programación, utilizando el lenguaje Scratch, de uno de los retos del nivel 2 del juego a fin de dejar evidencia de su factibilidad.

Así, todos los objetivos planteados para este estudio fueron alcanzados de forma satisfactoria, propiciando incluso la aproximación a un prototipo que permitiera visualizar el concepto del juego.

A manera de cierre, resulta pertinente resaltar que el desarrollo de esta investigación dejó clara la importancia de la enseñanza en todos los ámbitos, en este caso en el campo de la salud, viendo al paciente como un aprendiz. Asimismo, la necesidad imperativa de nuevas formas de comunicación e intervención instruccional, bien planificadas y correctamente fundamentadas desde el punto de vista pedagógico, para lograr el aprendizaje en los pacientes. Estas nuevas intervenciones deben estar vinculadas con los últimos avances en tecnología, la lingüística y con las metodologías que se están aplicando a nivel mundial, como es el uso de los videojuegos como mediadores y facilitadores del proceso de enseñanza y aprendizaje, para que sean motivadoras y significativas.

\section{RECOMENDACIONES.}

A partir de la experiencia obtenida durante el desarrollo de este estudio, se precisan las siguientes recomendaciones:

- Establecer alianzas con organizaciones involucradas con la divulgación de información acerca de la Enfermedad Celiaca para que con un equipo multidisciplinario integrado por nutricionistas, programadores y médicos especialistas, se pueda desarrollar este recurso de manera óptima.

- Para el desarrollo de este juego se recomienda el uso de un lenguaje de programación que permita la conexión con bases de datos debido a que es muy importante la conexión entre las actividades y los niveles. Las actividades son prelatorias entre sí, por lo cual es necesario que si el participante se equivoca pueda volver a intentarlo partiendo del punto en donde había quedado y no desde el inicio. 
Santos Rivera, J. M. y Navas Piñate, E. E. Juego de vídeo a partir de la metodología gamebased learning como medio de comunicación para la formación de hábitos alimentarios en niños con enfermedad celíaca

- Una vez desarrollado el recurso se sugiere su ampliación y actualización periódica incluyendo nuevos contenidos y actividades para la inclusión de nuevos niveles que permitan profundizar un poco más y reforzar los conocimientos obtenidos, así como mantener la jugabilidad del recurso, esta última, una característica crucial para mantener la motivación del jugador.

- La versatilidad del diseño de este videojuego permite su extrapolación a otros tópicos dentro del campo de la salud. En este sentido, se recomienda el ajuste de los contenidos con el fin de utilizarlo como recurso para la enseñanza de hábitos de estilo de vida saludable que incluyan otros aspectos además de la alimentación y que estén adaptados al contexto venezolano actual.

\section{REFERENCIAS}

Lenguaje de programación Scratch. (2019). Academy Pop. Recuperado de https://www.youtube.com/watch?v=rtQinc0anC8

Aznar, M. (2009). Repercusiones psicológicas de la Enfermedad Celiaca en niños y sus familias (Tesis Doctoral). Universidad Autónoma de Madrid, España. Recuperado de https://repositorio.uam.es/bitstream/handle/10486/3982/27648 aznar bola\%c3\% b10 margarita.pdf?sequence $=1$ \&isAllowed $=y$

Bravo, F. y Paz, M. (2011). Adherencia e impacto de la dieta sin gluten en niños con enfermedad celiaca. Revista Chilena de Pediatría, 82(3), 191- 197. doi: $10.4067 / \mathrm{S} 0370-41062011000300003$

Cabrera, M., González, J., Gutierrez, F., Paderewski, P., \& Padilla, N. (2008). Diseño de Videojuegos Educativos Multijugador. Una Visión desde el Aprendizaje Colaborativo. Presentado en IX Congreso Internacional Interacción, Albacete, España. Recuperado de http://www.citafgsr.org/cita/activos/texto/wfgsr articulo 0126 1051.pdf

Cabrera, M., González, J., Gutierrez, F., Paderewski, P y Padilla, N. (2009). Diseño de videojuegos colaborativos y educativos centrado en la jugabilidad. IEEE-RITA, 4(3),191-198. Recuperado de http://rita.det.uvigo.es/200908/uploads/IEEERITA.2009.V4.N3.A4.pdf

Contreras, R. (2016). Juegos Digitales y Gamificación aplicados en el ámbito de la Educación. RIED. Revista Iberoamericana de Educación a Distancia, 19(2), 27-33. doi: $10.5944 /$ ried.19.2.16143

Fabiano, F., Lista, D., Torres, J., y Urquiola, A. (2013). Primer estudio de prevalencia de la condición celíaca en Venezuela. Gen, 674), 203- 207. Recuperado de: http://www.genrevista.org/index.php/GEN/article/view/163 
Santos Rivera, J. M. y Navas Piñate, E. E. Juego de vídeo a partir de la metodología gamebased learning como medio de comunicación para la formación de hábitos alimentarios en niños con enfermedad celíaca

Israel, M. (2017). Game-based learning and gamification: Guidance from the experts [Aprendizaje basado en juegos y gamificación: Guía desde los expertos\archive PDF]. Recuperado de http://micheleisrael.com/wp-content/uploads/2017/11/Game -Based-Learning-Gamification.pdf

Monrroy, A. (2009, octubre 23). Conectar Scratch con base de datos. Mensaje publicado en: https://scratcharchive.asun.co/forums/viewtopic. php?id=24750

Ministerio de Salud de Argentina (2014). Cuadernillo para Nutricionistas. Manejo Nutricional de la Enfermedad Celiaca. Buenos Aires, Argentina. Recuperado de http://www.msal.gob.ar/celiacos/pdf/2014-01-22 guia-nutricionistas.pdf

Polanco, I. y Ribes, C. (2010). Enfermedad Celíaca. En Sociedad Española de Gastroenterología, Hepatología y Nutrición Pediátrica (Eds.) Protocolos DiagnóticoTerapéuticos de Gastroenterología, Hepatología y Nutrición Pediátrica. (pp. 3746). España: Ergón S.A. Recuperado de https://www.aeped.es/sites/default/files/documentos/celiaquia.pdf

Rojas, F., Silva, E., Hernández de Silva, A. (2013). Diseño y Desarrollo de Material Instruccional Computarizado. Herramientas TIC Aplicadas a la Educación. Universidad Simón Bolívar.

Technoguyx. (2009, octubre 20). Conectar Scratch con base de datos. Mensaje publicado en: https://scratcharchive.asun.co/forums/viewtopic .php?id=24750

Universidad Pedagógica Experimental Libertador (2016). Manual de Trabajos de Grado de Especialización y Maestría y Tesis Doctorales (5.a ed.). Caracas, Venezuela: Fondo Editorial de la Universidad Pedagógica Experimental Libertador. Recuperado de https://es.scribd.com/document/330024419/Manual-UPEL-2016pdf

\section{AUTORES}

\section{Jeanín M. Santos Rivera}

Licenciada en Nutrición y Dietética (UCV), Especialista en Tecnología, Aprendizaje y Conocimiento (UNIMET). Profesora asistente de la Universidad Metropolitana, Caracas, Venezuela, de las asignaturas de Arte y Ciencia de la Gastronomía, Educación Nutricional y Tecnologías para el Aprendizaje.

jsantos@unimet.edu.ve.

\section{Elvira E. Navas Piñate}

Ingeniero en Computación (USB), Especialista en Informática Educativa (USB), Doctora en Investigación en Didáctica y Organización de Instituciones Educativas (US). Profesora titular de la Universidad Metropolitana, Caracas, Venezuela, de las asignaturas de la línea de Programación de Computadoras y de la Tecnología Educativa. Decana de la Facultad de Ciencias y Artes. 
Santos Rivera, J. M. y Navas Piñate, E. E. Juego de vídeo a partir de la metodología gamebased learning como medio de comunicación para la formación de hábitos alimentarios en niños con enfermedad celíaca

enavas@unimet.edu.ve. 